\title{
CHRONIC KIDNEY DISEASE
}

\section{Reduced kidney function and abnormal cardiac structure}

\begin{abstract}
Abnormalities in left ventricular structure but not function precede the onset of clinical heart failure (HF) in individuals with chronic kidney disease (CKD), say researchers of a new study. Meyeon Park and colleagues found a significant association between renal function and abnormal left ventricular geometry across varying levels of renal dysfunction, but only minimal or no association with diastolic and systolic function. "Our findings ... offer additional insight into the severity of cardiac disease with lower levels of kidney function", state the authors in their paper.
\end{abstract}

\section{《Significant associations between eGFR and LVH were identified... 77}

Previous studies have identified associations between kidney function and structural cardiac changes in patients without HF, but the association of varying degrees of kidney dysfunction with changes in cardiac structure and function is unclear. Park et al. therefore evaluated the associations of different stages of estimated glomerular filtration rate (eGFR) with changes in cardiac structure and function in 3,487 participants of the Chronic Renal Insufficiency Cohort (CRIC) Study with established CKD and no known HF.

The researchers found that the prevalence of left ventricular hypertrophy (LVH) increased with decreasing eGFR as assessed by cystatin C. LVH was identified in $32 \%, 48 \%, 57 \%$, and $75 \%$ of individuals with eGFR $\geq 60 \mathrm{ml} / \mathrm{min} / 1.73^{2}, 45-59 \mathrm{ml} /$ $\mathrm{min} / 1.73^{2}, 30-44 \mathrm{ml} / \mathrm{min} / 1.73^{2}$, and $<30 \mathrm{ml} / \mathrm{min} / 1.73^{2}$, respectively. Significant associations between eGFR and LVH were identified across all categories of kidney dysfunction after adjustment for demographic factors, with stronger associations at lower levels of kidney function. After adjustment for comorbid conditions and markers of mineral metabolism, the association between eGFR and LVH remained significant for eGFR categories $<30 \mathrm{ml} / \mathrm{min} / 1.73^{2}$ and $30-44 \mathrm{ml} / \mathrm{min} / 1.73^{2}$. Associations between eGFR and left ventricular mass and abnormal left ventricular geometry were also found, which remained significant after full adjustment only for individuals with eGFR $<30 \mathrm{ml} / \mathrm{min} / 1.73^{2}$.

Diastolic dysfunction was associated with eGFR across all levels of kidney dysfunction in demographic-adjusted models. However, after full adjustment this association remained significant only for eGFR categories $<30 \mathrm{ml} / \mathrm{min} / 1.73^{2}$ and $45-59 \mathrm{ml} / \mathrm{min} / 1.73^{2}$. No association between eGFR category and reduced systolic function was identified.

The researchers say that future studies will investigate the extent to which each of the structural and functional abnormalities predicts the onset of clinical HF in CRIC participants.

Susan J. Allison

Original article Park, M. et al. Associations between kidney function and subclinical cardiac abnormalities in CKD. J. Am. Soc. Nephrol. doi:10.1681/ASN.2012020145 